\title{
Analysis of Electric Torque in Single Machine- Infinite Bus System Using Phase Shifters
}

\author{
A. L. S. Pereira, P. B. de Araujo, C. T. Miasaki
}

\begin{abstract}
ResumoThe main idea of this work is based on the analysis of the electric torque through the acting of the PS in the power system, provided of a control for the compensation degree (PSC). A linear model of the single machine-infinite bus system is used with a PS installed (SMIB/PS system). The variable that represents the presence of $\mathrm{PS}$ in the net is associated to the phase displacement introduced in the terminal voltage of the synchronous machine by PS. For the input signals of the PSC are evaluated variations of the angular speed of the rotor, the current magnitude and the active power through the line where the PS is located. The simulations are accomplished to analyze the influence of the PS in the torque formation (synchronizing and damping), of the SMIB/PS system. The analysis are developed in the time and frequency domain.
\end{abstract}

Keywords - Electric Torque; FACTS devices; Phase Shifters; Dynamic Stability; Power System.

\section{INTRODUÇ̃̃O}

$\mathrm{N}$ A era digital são grandes os desafios das empresas do setor elétrico para produzir e fornecer, de maneira eficiente e segura, energia para seus consumidores. As principais barreiras a serem superadas são o rápido crescimento da demanda, a infra-estrutura inadequada, a obrigação de equilibrar crescimento de energia com proteção ambiental, a reestruturação e a desregulamentação do setor de energia e a falta de investimentos no setor elétrico [9]. Percebe-se que essas barreiras contribuem para que o sistema existente opere de maneira diferente do que havia sido concebido e, conseqüentemente, existe a necessidade de se adotar novas estratégias para sua operação e planejamento.

A operação e planejamento de um sistema de potência são em grande parte complexos devido as linhas de transmissão estarem sujeitas a limites térmicos e/ou de estabilidade, que restringem o nível de potência que pode ser transmitido com segurança. Tais limites criam gargalos em alguns pontos da rede de transmissão. Esses pontos não são fixos; eles se modificam de acordo com as alterações nos fluxos de potência resultantes do despacho de geração, das características da

Este trabalho foi parcialmente financiado pela Capes.

Os autores pertecem ao Departamento de Engenharia Elétrica da Universidade Estadual Paulista de Ilha Solteira (FEIS - UNESP). Ilha Solteira - SP CEP 15385-000. (e-mails: andspa@gmail.com; percival@dee.feis.unesp.br,miasaki@gmail.com) carga e das contingências. Os problemas gerados pela presença desses pontos de gargalo no sistema são: baixa capacidade de carregamento, necessidade de redespacho da geração, exigência de capacidade extra de geração ou importação de energia e pontos de operação não econômicos [8]. Diante destes fatos, torna-se cada vez mais importante controlar o fluxo de potência nas linhas de transmissão.

Com o advento de novas tecnologias, notadamente o surgimento dos controladores FACTS, ocorreu uma melhora no controle dos fluxos das linhas e, conseqüentemente, um aumento da capacidade de transmissão de potência [5],[9],[10],[11],[12]. Em outras palavras, esses controladores propiciam um maior grau de liberdade na operação dos sistemas elétricos de potência [21].

O principal objetivo deste trabalho é estudar a influência de transformadores defasadores (um dispositivo FACTS), no sistema elétrico quando este é submetido a pequenas perturbações em torno de um ponto de equilíbrio [3],[4],[6],[7],[14]. O estudo se baseia na análise do torque elétrico formado no sistema elétrico de potência através do transformador defasador

Um transformador defasador é um componente promissor do sistema de potência no contexto de controladores FACTS, pois o controle do ângulo de fase das tensões das barras do sistema é um meio efetivo para se direcionar o fluxo de potência em uma rede de transmissão em corrente alternada [3],[13],[20]. Outras conseqüências da instalação do TD num sistema de energia elétrica são a minimização de perdas ativas na transmissão e melhorias na estabilidade do sistema elétrico [5],[7],[14],[18].

Neste trabalho será estudada a influência do transformador defasador na formação de torque elétrico (decomposto em parcelas de sincronização e amortecimento), no sistema elétrico de potência, reduzido a um sistema máquina síncrona versus barra infinita. Como o foco dos estudos é a estabilidade a pequenas perturbações, para a modelagem deste sistema equivalente, será utilizando um modelo linear similar ao conhecido como de "Heffron \& Phillips", considerando a operação de um TD [4],[19].

Para se avaliar o comportamento destes torques simulações são apresentadas e a partir delas, os resultados obtidos são comentados.

\section{FoRMULAÇÃO MATEMÁTICA DO SISTEMA MBI COM}




\section{TRANSFORMADOR DEFASADOR}

Normalmente, para facilitar os estudos iniciais de sistemas de potência de grande porte (sistemas reais, com vários geradores e várias linhas de transmissão), utiliza-se um sistema com apenas uma máquina que atua contra uma barra infinita [12]. Este sistema de potência, mais simples, é considerado a primeira etapa de estudos e tem como vantagem a possibilidade de fornecer informações que podem contribuir para a compreensão de sistemas de potência reais.

O sistema máquina síncrona versus barra infinita com um transformador defasador instalado (sistema MBI/TD), quando do estudo da estabilidade a pequenas perturbações, pode ser representado por um modelo linear cujas equações são mostradas na seqüência [4],[19].

$$
\begin{aligned}
& \Delta \stackrel{\circ}{\delta}=\omega_{0} \Delta \omega \\
& \circ \\
& 2 H \Delta \omega=\Delta T_{m}-\Delta T_{e}-D \Delta \omega \\
& T_{d 0^{\prime} \Delta e_{q}^{\prime}}^{\stackrel{\circ}{\prime}}=\Delta e_{f d}-K_{4} \Delta \delta-\frac{1}{K_{3}} \Delta e_{q}^{\prime}-K_{9} \Delta \phi \\
& \circ \\
& T_{r} \Delta e f d=-K_{r} \Delta v_{t}-\Delta e_{f d}+K_{r} \Delta v_{r e f} \\
& \Delta T_{e}=K_{1} \Delta \delta+K_{2} \Delta e_{q}^{\prime}+K_{8} \Delta \phi \\
& \Delta v_{t}=K_{5} \Delta \delta+K_{6} \Delta e_{q}^{\prime}+K_{7} \Delta \phi
\end{aligned}
$$

Com o conjunto (1), é possível representar o sistema $\mathrm{MBI} / \mathrm{TD}$ na forma de diagrama de blocos, como mostrado na Fig. 1.

Observe que este modelo é uma ampliação do modelo linear convencional de Heffron \& Phillips, largamente utilizado na literatura para estudos referentes à estabilidade a pequenas perturbações [2],[16],[17].

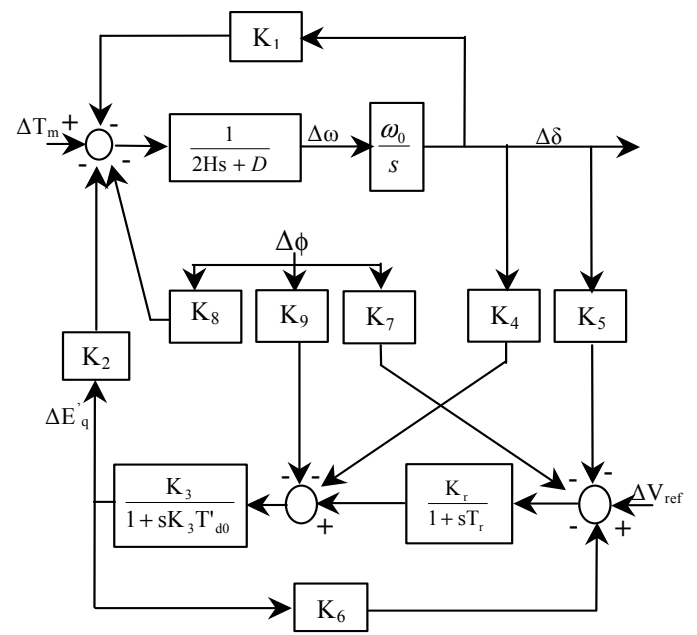

Fig. 1. Sistema MBI/TD.

\section{A. O TD com controle do grau de compensação ("CTD”)}

Para se introduzir uma dinâmica no grau de compensação, isto é, permitir que $\phi$ assuma valores de acordo com a necessidade do sistema de potência, é sugerido o sistema de controle mostrado na Fig. 2 [3].

No diagrama de blocos da Fig. 2, $\mathrm{K}_{\mathrm{t}}$ e $\mathrm{T}_{\mathrm{t}}$ representam o ganho e a constante de tempo do circuito de disparo do tiristor, respectivamente (neste trabalho $\mathrm{K}_{\mathrm{t}}$ será considerado unitário e $\mathrm{T}_{\mathrm{t}}$ nulo) [3]. O bloco de primeira ordem de ganho $\mathrm{K}_{\mathrm{aTD}}$ e constante de tempo $\mathrm{T}_{\mathrm{aTD}}$ representam a função de transferência do circuito necessário para a adequação da variável de entrada do dispositivo de controle. $\Delta \mathrm{y}(\mathrm{s})$ é o sinal de entrada para o CTD.

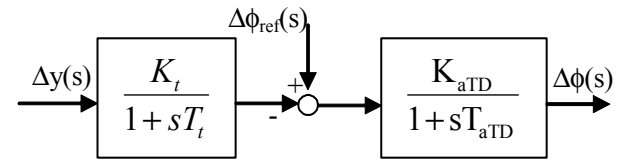

Fig. 2 Sistema de Controle do Grau de Compensação do TD (CTD).

Com as considerações anteriores, o CTD pode ser representado como na Fig. 3 (considerando $\Delta \phi_{\text {ref }}(\mathrm{s})=0$ ), que define o modelo a ser utilizado neste trabalho.

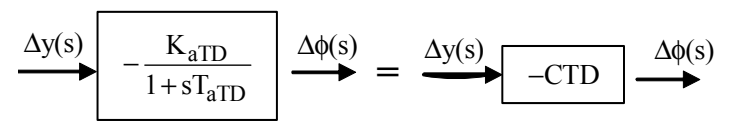

Fig. 3. Sistema de Controle do Grau de Compensação do TD (CTD) Resultante.

\section{CONTRIBUIÇÃO DO TD NA FORMAÇÃO DE TORQUE ELÉTRICO NO SISTEMA ELÉTRICO DE POTÊNCIA}

A influência do CTD no sistema elétrico de potência será avaliada através dos torques formados pelo TD. Para se analisar a formação e o comportamento destes torques, três sinais distintos são aplicados à entrada do controle do TD [1], [3], sendo eles as variações da velocidade angular do rotor $(\Delta \omega)$, as variações da corrente elétrica $(\Delta \mathrm{I})$ e as variações da potência elétrica $\left(\Delta \mathrm{P}_{\mathrm{e}}\right)$.

Considerando o modelo ampliado de Heffron \& Phillips (Fig. 1), e fazendo as devidas manipulações, é possível analisar os diferentes "caminhos" para formação de torque elétrico, pela atuação do TD, como mostra a Fig. 4.

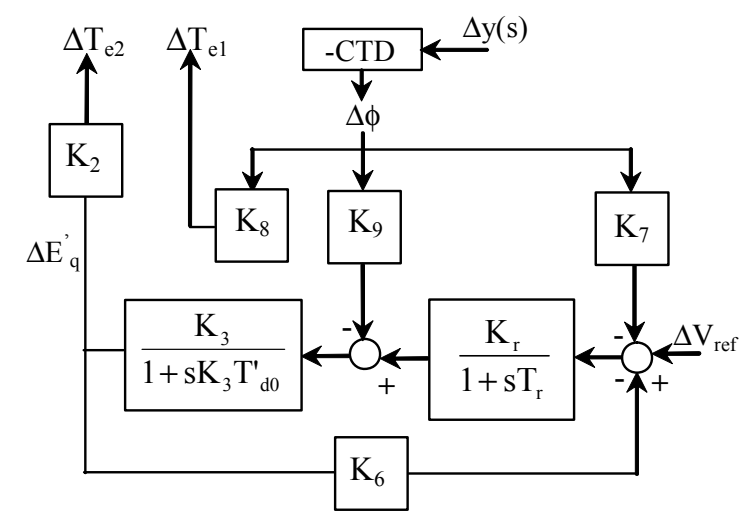

Fig. 4 Caminhos para Formação de Torque Elétrico através do TD.

Como se pode observar na Fig. 4, existem duas parcelas de torque elétrico formadas pela contribuição do $\mathrm{TD}\left(\Delta \mathrm{T}_{\mathrm{e} 1}\right.$ e $\Delta \mathrm{T}_{\mathrm{e} 2}$ 
- equações (2) e (3)), sendo que o torque elétrico total formado $\left(\Delta \mathrm{T}_{\mathrm{eTD}}\right)$ é mostrado na equação (4).

$$
\begin{aligned}
& \Delta T_{e 1}=K_{8} \Delta \phi=-K_{8} C T D \Delta y \\
& \Delta T_{e 2}=K_{2} \Delta E_{q}^{\prime} \\
& \Delta T_{e P S}=-K_{8} C T D \Delta y+K_{2} \Delta E_{q}^{\prime}
\end{aligned}
$$

Uma vez definido o sinal de entrada para o CTD $(\Delta y)$, o torque formado pode ser decomposto em parcelas de amortecimento e sincronização.

\section{A. Entrada $\Delta \omega$ (variações da velocidade do rotor)}

Considerando $\Delta y(\mathrm{~s})=\Delta \omega(\mathrm{s})$, a manipulação do diagrama de blocos da Fig. 4 fornece a expressão do torque elétrico formado no sistema de potência através do TD (equação (5)).

$$
\begin{aligned}
& \Delta T_{e T D}=F_{\omega}(s) \Delta \omega \\
& F_{\omega}(s)=\left[-K_{8}+\frac{K_{2} B\left(K_{9}+R A T K_{7}\right)}{1+B R A T K_{6}}\right] C T D
\end{aligned}
$$

$\mathrm{Na}$ equação (5), apenas para facilidade de notação, $\mathrm{B}=\mathrm{K}_{3} /\left(1+\mathrm{sK}_{3} \mathrm{~T}^{\prime}{ }_{\mathrm{d} 0}\right)$ e RAT é a função de transferência que representa o regulador automático de tensão.

Para uma dada freqüência $\omega$ (isto é, fazendo-se $s=j \omega$ ), a equação (5) pode ser decomposta em parcela de torque elétrico em fase com as variações do ângulo interno $\Delta \delta$ (torque de sincronização) e parcela de torque elétrico em fase com as variações da velocidade angular do rotor $\Delta \omega$ (torque de amortecimento), conforme a equação (6), onde $K_{\mathrm{S} \omega}$ e $K_{\mathrm{D} \omega}$ são coeficientes de torques de sincronização e amortecimento produzidos pelo TD utilizando-se como entrada para o CTD as variações da velocidade angular do rotor $(\Delta \omega)$, respectivamente.

$$
\begin{aligned}
& \Delta T_{e T D}=K_{S \omega} \Delta \delta+K_{D \omega} \Delta \omega \\
& K_{s \omega}=-\frac{\omega}{\omega_{0}}\left\{\operatorname{Im}\left[F_{\omega}(j \omega)\right]\right\} \\
& K_{D \omega}=\left\{\operatorname{Re} a l\left[F_{\omega}(j \omega)\right]\right\}
\end{aligned}
$$

Para se avaliar como se comportam os coeficientes $\mathrm{K}_{\mathrm{S} \omega} \mathrm{e}$ $\mathrm{K}_{\mathrm{D} \omega}$ quando da variação do carregamento do sistema $\mathrm{MBI} / \mathrm{TD}$, foram realizadas simulações cujos resultados são mostrados na Fig. 5. As condições de simulação foram tensão terminal igual a $1(\mathrm{pu})$ e potência elétrica $\left(\mathrm{P}_{\mathrm{e}}\right)$ variando entre $0.1 \mathrm{e} 1.0(\mathrm{pu})$, com fator de potência 0.85 indutivo. Os valores dos parâmetros do sistema MBI/TD encontram-se no Apêndice A. Os coeficientes $K_{S \omega}$ e $K_{D \omega}$ foram calculados na freqüência natural do laço eletromecânico $\omega_{\mathrm{s}}$.

Observa-se da Fig. 5, que no sistema MBI/TD, a utilização de $\Delta \omega$ como entrada para o CTD produz praticamente torque de amortecimento, visto que $\mathrm{K}_{\mathrm{S} \omega}$ pode ser desprezado quando comparado com $\mathrm{K}_{\mathrm{D} \omega}$. Além disso, $\mathrm{K}_{\mathrm{D} \omega}$ aumenta com o aumento do carregamento do sistema. Este fato é interessante, pois em termos de estabilidade a pequenas perturbações os maiores problemas ocorrem para sistemas carregados, com amortecimento negativo. O pequeno valor de torque de sincronização negativo produzido através do TD não deve comprometer a integridade do sistema MBI/TD, pois as outras contribuições de torque de sincronização existentes (principalmente através de $\mathrm{K}_{1}$ ), deverão suprir esta deficiência.

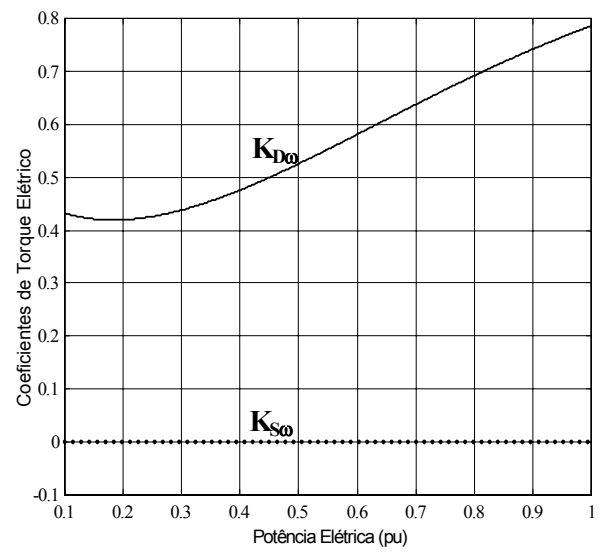

Fig. 5. Coeficientes $\mathrm{K}_{\mathrm{S} \omega}$ e $\mathrm{K}_{\mathrm{D} \omega}$.

\section{B. Entrada $\Delta I$ (variações da corrente elétrica)}

De maneira semelhante, considerando $\Delta \mathrm{y}(\mathrm{s})=\Delta \mathrm{I}(\mathrm{s})$, a manipulação do diagrama de blocos da Fig. 4 fornece a expressão do torque elétrico formado no sistema de potência através do TD, como na equação (7), onde as constantes $m_{1}$, $\mathrm{m}_{2}$ e $\mathrm{m}_{3}$ são definidas no Apêndice B.

$$
\begin{aligned}
& \Delta T_{e T D}=F_{I}(s) \Delta \delta \\
& F_{I}(s)=\left[K_{2} x-K_{8} C T D \frac{\left(m_{1}+m_{2} x\right)}{\left(1+C T D m_{3}\right)}\right] \\
& x=\frac{B\left(K_{7} R A T+K_{9}\right) C T D m_{1}}{\left[\left(1+K_{6} B R A T\right)\left(1+C T D m_{3}\right)-B\left(K_{7} R A T+K_{9}\right) C T D m_{2}\right]}
\end{aligned}
$$

De forma análoga à realizada para entrada $\Delta \omega$, são definidos os coeficientes de torques de sincronização $\left(\mathrm{K}_{\mathrm{SI}}\right)$ e amortecimento $\left(\mathrm{K}_{\mathrm{DI}}\right)$, quando a entrada para o CTD são os desvios de corrente elétrica, como na equação (8).

$$
\begin{aligned}
& \Delta T_{e T D}=K_{S I} \Delta \delta+K_{D I} \Delta \omega \\
& K_{S I}=\left\{\operatorname{Re} a l\left[F_{I}(j \omega)\right]\right\} \\
& K_{D I}=\frac{\omega_{0}}{\omega}\left\{\operatorname{Im}\left[F_{I}(j \omega)\right]\right\}
\end{aligned}
$$

Novamente foram realizadas simulações nas mesmas condições anteriores e o comportamento dos coeficientes $\mathrm{K}_{\mathrm{SI}} \mathrm{e}$ 
$\mathrm{K}_{\mathrm{DI}}$ são mostrados na Fig. 6.

Da Fig. 6, conclui-se que no sistema MBI/TD, a utilização de $\Delta \mathrm{I}$ como entrada para o CTD produz maior parcela de torque de amortecimento que de sincronização $\left(\mathrm{K}_{\mathrm{DI}}>\mathrm{K}_{\mathrm{SI}}\right)$. Ainda para este caso, o sinal de entrada $\Delta \mathrm{I}$ faz com que surja no sistema (apesar de pequena), uma contribuição negativa de torque de sincronização nas situações de baixo carregamento (para $\mathrm{P}_{\mathrm{e}}$ variando entre 0.1 à $0.18(\mathrm{pu})$ ). Além disso, tanto $\mathrm{K}_{\mathrm{SI}}$ quanto $\mathrm{K}_{\mathrm{DI}}$ aumentam de acordo com o aumento do carregamento do sistema. Observe também que em termos quantitativos, os coeficientes de torque de amortecimento obtidos com a entrada $\Delta \mathrm{I}$ são bem maiores que os obtidos com a entrada $\Delta \omega$ (este fato se deve à presença do termo $\omega_{0} / \omega$ presente quando da entrada $\Delta \mathrm{I}$ para se fazer a conversão de torque para o eixo $\Delta \omega)$.

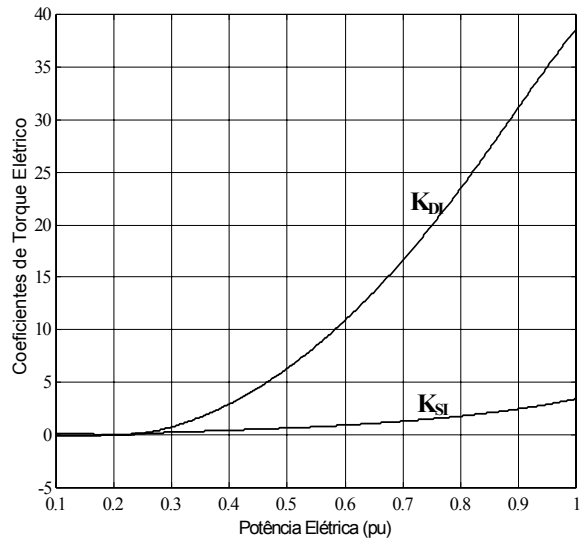

Fig. 6. Coeficientes $\mathrm{K}_{\mathrm{SI}}$ e $\mathrm{K}_{\mathrm{DI}}$ -

\section{Entrada $\Delta P_{e}$ (variações da potência elétrica)}

Considerando agora $\Delta \mathrm{y}(\mathrm{s})=\Delta \mathrm{P}_{\mathrm{e}}(\mathrm{s})$, a redução do diagrama de blocos da Fig. 4, fornece a expressão do torque elétrico formado no sistema de potência através do TD, como na equação (9).

$$
\begin{aligned}
& \Delta T_{e T D}=F_{P e^{(s) \Delta \delta}} \\
& F_{P e^{(s)}}=\left[K_{2} x-K_{8} C T D \frac{\left(K_{1}+K_{2} x\right)}{\left(1+C T D K_{8}\right)}\right] \\
& x=\frac{B\left(K_{7} R A T+K_{9}\right) C T D K_{1}}{\left.\left(1+K_{6} B R A T\right)\left(1+C T D K_{8}\right)-B\left(K_{7} R A T+K_{9}\right) C T D K_{2}\right]}
\end{aligned}
$$

Para este caso (a entrada para o CTD são os desvios de potência elétrica $\left.-\Delta \mathrm{P}_{\mathrm{e}}\right)$, os coeficientes de torque de sincronização $\left(\mathrm{K}_{\mathrm{SPe}}\right)$ e torque de amortecimento $\left(\mathrm{K}_{\mathrm{DPe}}\right)$ são dados na equação (10).

Os resultados das simulações realizados para este caso são mostrados na Fig. 7.

Observando o comportamento dos coeficientes de torque elétrico $\mathrm{K}_{\mathrm{DPe}}$ e $\mathrm{K}_{\mathrm{SPe}}$ da Fig. 7, pode-se concluir que a utilização da potência elétrica como entrada para o CTD no sistema
MBI/TD irá produzir torque de amortecimento superior ao torque de sincronização nas situações de carregamentos elevados, sendo que ambos os torques aumentam com o aumento do carregamento do sistema.

$$
\begin{aligned}
& \Delta T_{e T D}=K_{S P e} \Delta \delta+K_{D P e} \Delta \omega \\
& K_{S P e}=\left\{\operatorname{Re} a l\left[F_{P e}(j \omega)\right]\right\} \\
& K_{D P e}=\frac{\omega_{0}}{\omega}\left\{\operatorname{Im}\left[F_{P e}(j \omega)\right]\right\}
\end{aligned}
$$

Ainda com relação a este caso, outro fato a ser ressaltado é que para o carregamento variando de $0.1 \mathrm{(pu})$ até por volta de 0.3 (pu) (baixos carregamentos), existe uma contribuição de torque de amortecimento negativo $\left(\mathrm{K}_{\mathrm{DPe}}<0\right)$. Este amortecimento negativo introduzido através do TD não deve comprometer a integridade do sistema de potência nesta faixa de operação, pois a atuação do regulador automático de tensão introduz amortecimento positivo (através do parâmetro $\mathrm{K}_{5}$, positivo para baixos carregamentos), e proporcional ao ganho do regulador [2]. Além disso, os problemas relacionados à estabilidade ocorrem para sistemas bastante carregados e como se pode observar da Fig. 7, nestas condições o coeficiente de torque de amortecimento é positivo.

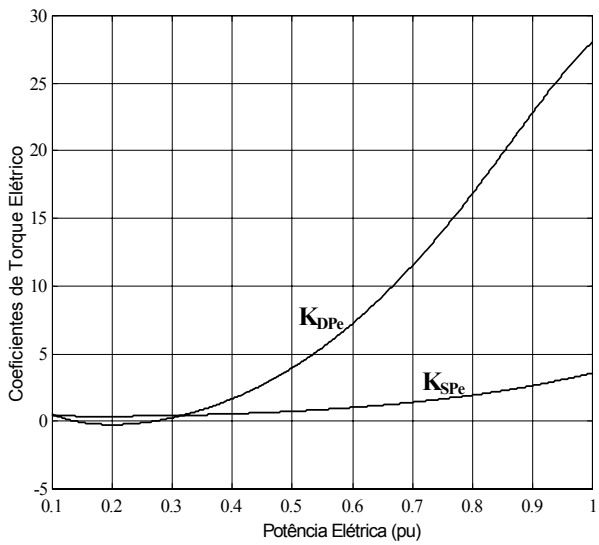

Fig. 7. Coeficientes $\mathrm{K}_{\mathrm{SPe}}$ e $\mathrm{K}_{\mathrm{DPe}}$.

\section{ANÁlISE DINÂMICA do SISTEMA MBI/TD}

Foram realizadas simulações no domínio do tempo para se avaliar a influência dos sinais de entrada do CTD no amortecimento das oscilações do sistema MBI/TD. O distúrbio aplicado foi uma variação em degrau de $0.05(\mathrm{pu})$ no torque mecânico de entrada $\left(\Delta \mathrm{T}_{\mathrm{m}}\right)$. $\mathrm{O}$ carregamento considerado foi de $0.8(\mathrm{pu})$, com fator de potência 0.85 indutivo. A defasagem inicial introduzida pelo TD no sistema de potência foi mantida em $20^{\circ}$ (os demais dados são mostrados no Apêndice A). As curvas referentes às variações da velocidade angular do rotor são mostradas na Fig. 8.

Como já era esperado da análise quantitativa dos coeficientes de torque de amortecimento, o CTD com entrada variações da corrente elétrica $(\Delta \mathrm{I})$, é mais eficaz no amortecimento das oscilações do sistema MBI/TD, seguido 
pelos sinais potência elétrica e velocidade do rotor $\left(\Delta \mathrm{P}_{\mathrm{e}}\right.$ e $\Delta \omega$, respectivamente). Observe também que nos instantes iniciais da resposta, os sinais corrente elétrica e potência elétrica apresentam resultados semelhantes.

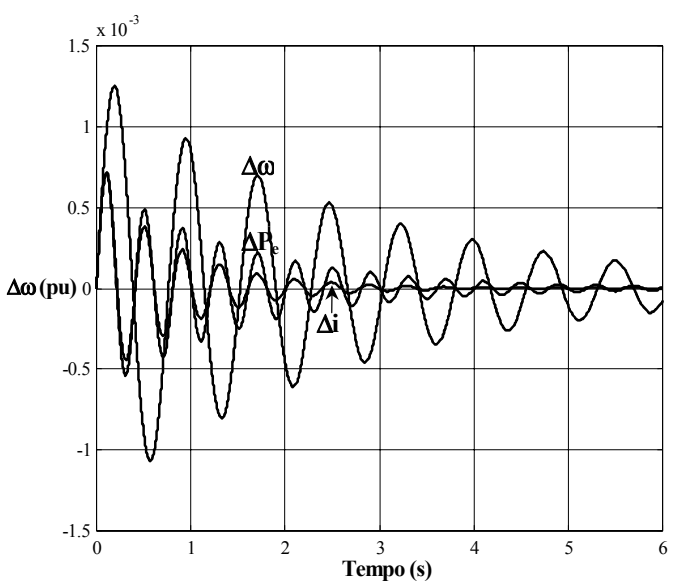

Fig. 8. Análise Dinâmica do Sistema MBI/TD.

Para uma análise no domínio da freqüência, considere a Tabela I, onde os modos eletromecânicos de oscilação, com freqüências em torno de $9 \mathrm{rad} / \mathrm{s}$ (típica de modo local), são mostrados na coluna 4, linhas 2, 7 e 10.

Além disso, quando são utilizados como entradas do CTD os sinais $\Delta \mathrm{I}$ e $\Delta \mathrm{P}_{\mathrm{e}}$, outros modos de oscilação surgem, com freqüências em torno de $16 \mathrm{rad} / \mathrm{s}$ (coluna 4, linhas 6 e 9). Estes modos são os que apresentam menor amortecimento no sistema MBI/TD considerado.

TABELA I

AUTOVALORES DO SISTEMA MBI/TD

\begin{tabular}{|l|l|l|c|}
\hline Entradas & Autovalores & $\xi$ & $\omega_{\mathrm{n}}$ \\
\hline \multirow{4}{*}{$\Delta \omega$} & -999.8507 & - & - \\
\cline { 2 - 4 } & $-0.3700 \pm 8.292 \mathrm{i}$ & 0.0445 & 8.30 \\
\cline { 2 - 4 } & -11.955 & - & - \\
\cline { 2 - 4 } & -8.0050 & - & - \\
\hline \multirow{4}{*}{$\Delta \mathrm{P}_{\mathrm{e}}$} & -293.0152 & - & - \\
\cline { 2 - 4 } & $-0.6646 \pm 15.725 \mathrm{i}$ & 0.0422 & 15.739 \\
\cline { 2 - 4 } & $-9.5091 \pm 0.631 \mathrm{i}$ & 0.9978 & 9.530 \\
\hline \multirow{3}{*}{$\Delta \mathrm{I}$} & -294.7516 & - & - \\
\cline { 2 - 4 } & $-1.1694 \pm 15.814 \mathrm{i}$ & 0.0737 & 15.857 \\
\cline { 2 - 5 } & $-9.1387 \pm 2.3304 \mathrm{i}$ & 0.9689 & 9.4311 \\
\hline
\end{tabular}

Da análise dos coeficientes de amortecimento ( $\xi$ - coluna 3 , linhas 2, 6 e 9), observa-se que a utilização do sinal de entrada $\Delta \mathrm{I}$ fornece o melhor desempenho (maior valor de $\xi$ para os modos menos amortecidos).

Outra observação a ser feita com relação à Tabela I é que observando as colunas 3 e 4 (coeficiente de amortecimento $\xi$ e freqüência natural $\omega_{\mathrm{n}}$ ), verifica-se que o TD é eficaz tanto para modos de oscilação eletromecânico (coluna 4 - linhas 2, 7 e 10), quanto para os outros modos de oscilação (coluna 4 linhas 6 e 9 ).

\section{CONClusões}

Neste trabalho foram apresentados estudos a respeito da inclusão do dispositivo FACTS TD no sistema de potência máquina síncrona ligada a uma barra infinita e sua influência na estabilidade a pequenas perturbações.

A partir de um modelo linear do sistema MBI com um TD em operação, foi introduzido um sistema de controle para o grau de compensação e se estudou a influência do sinal de entrada deste controle no torque elétrico formado através do TD. As entradas consideradas foram as variações da velocidade angular do rotor $(\Delta \omega)$, as variações da corrente elétrica $(\Delta \mathrm{I})$ e as variações da potência elétrica $\left(\Delta \mathrm{P}_{\mathrm{e}}\right)$.

Com o objetivo de avaliar o comportamento destes torques no sistema MBI/TD foram realizadas simulações onde se analisou os coeficientes de torque elétrico de sincronização e amortecimento em função do carregamento do sistema.

Das simulações verificou-se que o sinal de entrada referente às variações da corrente elétrica $(\Delta \mathrm{I})$ quando aplicado à entrada do CTD, proporcionou a maior contribuição no que se refere a torque de amortecimento, nas situações de altos carregamentos. Ainda para este caso, a contribuição de torque de sincronização foi negativa (para $P_{e}$ variando entre $0.1(\mathrm{pu})$ e $0.18(\mathrm{pu})$ ), mas foi aumentando de acordo com o aumento do carregamento do sistema.

Com relação ao sinal do controle referente às variações da potência elétrica $\left(\Delta \mathrm{P}_{\mathrm{e}}\right)$, observou-se que este forneceu torque elétrico de sincronização positivo em toda a faixa de carregamentos considerada. Em se tratando de torque de amortecimento, para o sistema elétrico operando com carregamentos baixos este foi negativo, tornando-se positivo para carregamentos elevados.

No que se refere ao torque de sincronização, os sinais $\Delta \mathrm{I}$ e $\Delta \mathrm{P}_{\mathrm{e}}$ apresentam praticamente a mesma contribuição, podendo ser desprezada a contribuição de sincronização proveniente da entrada $\Delta \omega$, quando comparada com as provenientes dos outros sinais.

A utilização das variações da velocidade angular do rotor $(\Delta \omega)$ como sinal de entrada para o CTD produz praticamente torque de amortecimento, visto que $\mathrm{K}_{\mathrm{S} \omega}$ é bem pequeno quando comparado com $\mathrm{K}_{\mathrm{D} \omega}$. Outro fato a ser ressaltado é que dentre os sinais aplicados ao controle, foi o que menos contribuiu na formação de torque elétrico no sistema MBI/TD (este fato pode ser explicado devido ao baixo ganho utilizado no CTD).

Por meio de simulações no domínio do tempo, concluiu-se pela maior contribuição do sinal $\Delta \mathrm{I}$ no amortecimento das oscilações do sistema elétrico de potência. Observou-se também que os sinais corrente elétrica e potência elétrica, ao contrário do sinal velocidade angular do rotor, excitaram outros modos de oscilação, além do de modo local. Mesmo assim, os sinais $\Delta \mathrm{I}$ e $\Delta \mathrm{P}_{\mathrm{e}}$ se mostraram mais eficientes no amortecimento dos modos oscilatórios que o sinal $\Delta \omega$.

\section{APÊNDICE A}

Dados do Sistema Exemplo (adaptação de [15]) (em 
valores pu, a não ser quando especificado).

Gerador:

$\mathrm{x}^{\prime}{ }_{\mathrm{d}}=0.245 \quad \mathrm{x}_{\mathrm{d}}=1.7 \quad \mathrm{x}_{\mathrm{q}}=1.64 \quad \mathrm{~T}_{\mathrm{d} 0}=5.9(\mathrm{~s})$

$\mathrm{D}=0.0 \quad \mathrm{H}=2.37(\mathrm{~s})$

Linha de Transmissão:

$\mathrm{R}_{\mathrm{L}}=0.0 \quad \mathrm{X}_{\mathrm{L}}=0.4$

Regulador de Tensão: representado por um bloco de primeira ordem com ganho $\mathrm{K}_{\mathrm{r}}$ e constante de tempo $\mathrm{T}_{\mathrm{r}}$.

$\mathrm{K}_{\mathrm{r}}=50.0 \quad \mathrm{~T}_{\mathrm{r}}=0.01(\mathrm{~s})$

Controle do TD (CTD):

$\mathrm{K}_{\mathrm{aTD}}=0.8 \quad \mathrm{~T}_{\mathrm{aTD}}=0.001(\mathrm{~s})$

\section{APÊNDICE B}

Desvios da corrente terminal $\Delta \mathrm{i}_{\mathrm{t}}$ :

$$
\tilde{i}_{t}=i_{d}+j i_{q} \rightarrow\left|\tilde{i}_{t}\right|=i_{t}=\sqrt{i_{d}^{2}+i_{q}^{2}}
$$

Para pequenos desvios:

$$
\begin{aligned}
\Delta \mathrm{i}_{\mathrm{t}} & =\mathrm{m}_{1} \Delta \delta+\mathrm{m}_{2} \Delta \mathrm{e}_{\mathrm{q}}+\mathrm{m}_{3} \Delta \phi \\
m_{1} & =\frac{i_{d}}{i_{t}} \frac{\partial i_{d}}{\partial \delta}+\frac{i_{q}}{i_{t}} \frac{\partial i_{q}}{\partial \delta} \\
m_{2} & =\frac{i_{d}}{i_{t}} \frac{\partial i_{d}}{\partial e_{q}^{\prime}} \\
m_{3} & =\frac{i_{d}}{i_{t}} \frac{\partial i_{d}}{\partial \phi}+\frac{i_{q}}{i_{t}} \frac{\partial i_{q}}{\partial \phi}
\end{aligned}
$$

\section{REFERÊNCIAS}

[1] A. D. Del Rosso, C. A. Canizares, V. M. Doña, "A study of TCSC controller design for power system stability improvement", IEEE Transactions on Power Systems, v. 18, n. 4, pp. 1487-1496, 2003.

[2] F. P. Demello, C. Concórdia, "Concepts of Synchronous Machine Stability as Affected by Excitation Control", IEEE Transactions on Power Apparatus and Systems, v. 88, n. 4, pp. 316-329, 1969.

[3] K. Xing, G. Kusic, "Application of Thyristor-controlled Phase Shifter to Minimize Real Power Losses and Augment Stability of Power Systems", IEEE Transactions on Energy Conversion, v. 3, n. 4, pp. 792-798, 1988.

[4] H. F. Wang, F. J; Swift, "A Unified Model for the Analysis of FACTS Devices in Damping Power Oscillations. Part I: Single-machine Infinitebus Power Systems", IEEE Transactions on Power Delivery, v. 12, n. 2, pp. 945-946, 1997.

[5] IEEE FACTS TERMS \& DEFINITIONS TASK FORCE.: "Proposed Terms and Definitions for Flexible AC Transmission Systems (FACTS)", IEEE Transactions on Power Delivery, v. 12, n. 4, pp. 1848$1853,1997$.

[6] S. Gomes Jr., N. Martins, C. Portela, "Computing Small-Signal Stability Boundaries for Large-Scale Power Systems", IEEE Transactions on Power Systems, USA, n. PE-011PRS, p. xx-xx, 2003.

[7] Y. L. Kang, G. B. Shrestha, T. T. Lie, "Improvement of Power System Dynamic Performance with the Magnitude and Phase Angle Control of Static Phase Shifter", Electric Power Systems Research, n. 55, pp. 121$128,1999$.

[8] G. Nilson, J. Bahrman, "Power Flow Control". Panel Session on FACTS Changes Power System Control, IEEE/PES Winter Power Meeting, pp. $1-15,1994$

[9] G. Reed, J. Paserba, P. Salavantis, "The FACTS on Resolving Transmission Gridlock”, IEEE Power \& Energy Magazine, pp 41-46, setembro/outubro, 2003.
[10] E. H. Watanabe, P. G. Barbosa, K. C. Almeida \& G. N. Taranto, "Tecnologia FACTS: Tutorial," Revista Brasileira de Controle \& Automação, Vol. 9 No. 1, pp. 39-55, abril, 1998.

[11] A. B. Nassif, L. C. P. da Silva, V. F. da Costa, "Effects of the SVC and the STATCOM on Damping Power Systems Low Frequency Electromechanical Oscillations", IEEE LATIN AMERICA TRANSACTIONS, Vol. 2, No. 2, pp. 120-125, June 2004

[12] L. D. Colvara, "The Influence of the TCSC on Power System Transient Energy", IEEE LATIN AMERICA TRANSACTIONS, Vol. 3, No. 3, pp. 284-289, July 2005.

[13] R. Mihalic, U. Gabrijel , "Transient stability assessment of systems comprising phase-shifting FACTS devices by direct methods" International Journal of Electrical Power and Energy Systems, 26 (6), p.445-453, jul 2004.

[14] A. A. Hashmani, Y. Wang, and T. T. Lie, "Design and Application of a Nonlinear Coordinated Excitation and TCPS Controller in Power Systems", International Journal of Control, Automation, and Systems, vol. 3, no. 2, pp.346-354, 2005.

[15] P. Anderson, A. A. Fouad, Power System Control and Stability, $2^{\text {th }}$ edition, (A John Wiley \& Sons, INC., Publication), EUA, 2003, p. 658.

[16] P. Kundur, Power System Stability and Control, (Electrical Power Research Institute, MacGraw-Hill), EUA, 1994, p. 1167.

[17] Y. N. Yu, Electric Power System Dynamic, (Academic Press), EUA, 1983, p. 255.

[18] E. D. Festraits, L. D. Colvara, S. C. B. Araújo, "Estudo da Influência de Dispositivos FACTS na Estabilidade de Sistemas de Potência", Anais do XIV Congresso Brasileiro de Automática, Natal, RN, 2002.

[19] A. L. S. Pereira, M. S. Silva, P. B. Araújo, "Modelo Linear de Heffron \& Phillips com a inclusão do Defasador ("Phase Shifter" (PS))", Anais do $3^{\circ}$ Congresso Temático de Dinâmica e Controle da SBMAC, junho 2004

[20] M. Reza, W. L. Kling, "Solving Network Constraints (Congestions) by Investigating Impact of Phase Shifters on the Flows", Proceedings of the Second IASTED International Conference Power and Energy Systems, pp.189-193, junho 2002.

[21] Y. H. Song, A. T. Johns, Flexible AC Transmission Systems (FACTS), (The Institution of Electrical Engineers, TJ International Ltd.), England, United Kingdom, 1999, p. 592.

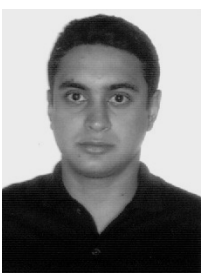

André Luiz Silva Pereira nasceu em Ilha Solteira (SP); em 2002 obteve o titulo de Engenheiro Eletricista pela Universidade Federal de Mato Grosso (UFMT). Em 2005 tornou-se mestre em Engenharia Elétrica pela Universidade Estadual Paulista de Ilha Solteira (UNESP-FEIS). Atualmente é aluno de Doutorado do programa de pós graduação em Engenharia Elétrica (PPGEE-FEIS). Sua área de interesse esta relacionada à Estabilidade Dinâmica de Sistemas de Energia Elétrica.

Percival Bueno de Araujo obteve o título de Engenheiro Eletricista na Universidade Estadual Paulista Júlio de Mesquita Filho, UNESP-FEIS em 1982; em 1988 tornou-se mestre em Engenharia Elétrica na Universidade Federal de Santa Catarina, UFSC. Em 1998, Doutor em Engenharia Elétrica na Universidade de São Paulo, USP. Atualmente é Professor Assistente Doutor da UNESP/FEIS, Ilha Solteira, Brasil. Sua principal área de interesse está relacionada à Estabilidade Dinâmica de Sistemas de Energia Elétrica.

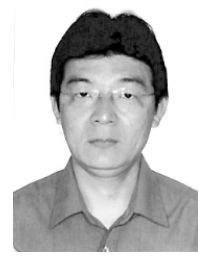

Celso Tadao Miasaki obteve o Titulo de Licenciatura em Matemática na UFMS/Três Lagoas-MS em 1993. Concluiu o Mestrado e Doutorado em Engenharia Elétrica na UNESP/Ilha Solteira-SP (Brazil) em 2002 e 2006, respectivamente. Atualmente é Professor do Departamento de Matemática da FUNEC/Santa Fé do Sul. Suas principais áreas de interesse são Planejamento de Sistemas de Transmissão e Dispositivos FACTS. 